\title{
Cost-Effectiveness of Initial Versus Delayed Lanreotide for Treatment of Metastatic Enteropancreatic Neuroendocrine Tumors
}

\author{
James I. Barnes, MD, MS',2; John K. Lin, MD1,2; Divya Gupta, MD³; Douglas K. Owens, MD, MS,2; \\ Jeremy D. Goldhaber-Fiebert, $\mathrm{PhD}^{2}$; and Pamela L. Kunz, MD
}

\begin{abstract}
Background: The Controlled Study of Lanreotide Antiproliferative Response in Neuroendocrine Tumors (CLARINET) trial showed prolonged progression-free survival in patients initially treated with lanreotide versus placebo. We evaluated the cost-effectiveness of upfront lanreotide versus active surveillance with lanreotide administered after progression in patients with metastatic enteropancreatic neuroendocrine tumors (NETs), both of which are treatment options recommended in NCCN Clinical Practice Guidelines in Oncology for Neuroendocrine and Adrenal Tumors. Methods: We developed a Markov model calibrated to the CLARINET trial and its extension. We based the active surveillance strategy on the CLARINET placebo arm. We calculated incremental cost-effectiveness ratios (ICERs) in dollars per quality-adjusted life-year (QALY). We modeled lanreotide's cost at $\$ 7,638$ per $120 \mathrm{mg}$ (average sales price plus $6 \%$ ), used published utilities (stable disease, 0.77; progressed disease, 0.61), adopted a healthcare sector perspective and lifetime time horizon, and discounted costs and benefits at $3 \%$ annually. We examined sensitivity to survival extrapolation and modeled octreotide long-acting release (LAR) $(\$ 6,183$ per $30 \mathrm{mg}$ ). We conducted one-way, multiway, and probabilistic sensitivity analyses. Results: Upfront lanreotide led to 5.21 QALYs and a cost of $\$ 804,600$. Active surveillance followed by lanreotide after progression led to 4.84 QALYs and a cost of $\$ 590,200$, giving an ICER of $\$ 578,500 / \mathrm{QALY}$ gained. Reducing lanreotide's price by $95 \%$ (to $\$ 370$ ) or $85 \%$ (to $\$ 1,128$ ) per $120 \mathrm{mg}$ would allow upfront lanreotide to reach ICERs of $\$ 100,000 /$ QALY or $\$ 150,000 /$ QALY. Across a range of survival curve extrapolation scenarios, pricing lanreotide at $\$ 370$ to $\$ 4,000$ or $\$ 1,130$ to $\$ 5,600$ per $120 \mathrm{mg}$ would reach ICERs of $\$ 100,000 / \mathrm{QALY}$ or $\$ 150,000 / \mathrm{QALY}$, respectively. Our findings were robust to extensive sensitivity analyses. The ICER modeling octreotide LAR is $\$ 482,700 / Q A L Y$ gained. Conclusions: At its current price, lanreotide is not cost-effective as initial therapy for patients with metastatic enteropancreatic NETs and should be reserved for postprogression treatment. To be cost-effective as initial therapy, the price of lanreotide would need to be lowered by $48 \%$ to $95 \%$ or $27 \%$ to $86 \%$ to reach ICERs of $\$ 100,000 / \mathrm{QALY}$ or $\$ 150,00 / \mathrm{QALY}$, respectively.
\end{abstract}

J Natl Compr Canc Netw 2020;18(9):1200-1209 doi: $10.6004 /$ jnccn.2020.7563

\footnotetext{
${ }^{1}$ VA Palo Alto Health Care System, Palo Alto, California; ${ }^{2}$ Center for Primary Care and Outcomes Research/Center for Health Policy, Department of Medicine, Stanford University School of Medicine, Stanford, California; and ${ }^{3}$ Department of Medicine, Stanford University, Stanford, California; and ${ }^{4}$ Department of Medical Oncology, Yale School of Medicine, New Haven, Connecticut.
}

\section{Background}

Incidence and prevalence of neuroendocrine tumors (NETs) have increased over time. Since the 1980s, incidence has increased by 6.4 times to 7 per 100,000 people in the United States, and 20-year limited-duration prevalence has increased by 8 times to $0.048 \% .^{1} \mathrm{Ap}$ proximately $61 \%$ of NETs arise from the gastrointestinal tract, and those that are grades 1 to 2 are relatively indolent, with median overall survival (OS) of approximately 1 decade. ${ }^{1}$

In asymptomatic patients with low-tumor-burden metastatic gastroenteropancreatic NETs, NCCN Clinical Practice Guidelines in Oncology (NCCN Guidelines) for Neuroendocrine and Adrenal Tumors ${ }^{2}$ recommend a somatostatin analog (lanreotide or octreotide) upfront or active surveillance with a somatostatin analog administered after disease progression as first-line therapy options for gastrointestinal NETs and as considerations for pancreatic NETs. Patients in the CLARINET trial (Controlled Study of Lanreotide Antiproliferative Response in Neuroendocrine Tumors) who received lanreotide upfront experienced prolonged progression-free survival (PFS) compared with those who received active surveillance (median, 32.8 vs $18 \mathrm{mo}$ ), without a statistically significant difference in OS. ${ }^{3}$ The CLARINET extension ${ }^{4}$ followed patients whose disease progressed on placebo and switched to lanreotide, showing a 14-month median PFS. Lanreotide is priced at $\$ 7,638$ per 28 days or $\$ 99,300$ per year (January 2020 Medicare Part B average sales price [ASP]-based maximum payment allowance), ${ }^{5}$ nearly doubling in inflation-adjusted terms over the past decade. ${ }^{6}$ Because of the indolent nature of NETs, patients often remain on therapy for several years before progression.

As NCCN moves toward incorporating affordability into its evidence base (NCCN Evidence Blocks), it is important to assess the value of recommended therapies. ${ }^{7}$ This study evaluated the cost-effectiveness of

See JNCCN.org for supplemental online content. 
upfront lanreotide versus active surveillance with lanreotide administered after disease progression for patients with grades 1 to 2 nonfunctioning gastroenteropancreatic NETs.

\section{Methods}

\section{Study Population}

We developed a discrete time semi-Markov model simulating a cohort of patients with advanced, well- or moderately differentiated, nonfunctioning, somatostatin receptor-positive enteropancreatic NETs of grade 1 or 2, $44.1 \%$ of whom have tumors of pancreatic origin. Our model followed these patients monthly over their remaining lifetimes.

\section{Treatment Strategies}

We evaluated 2 strategies: (1) upfront lanreotide and (2) active surveillance with lanreotide administered after disease progression ("active surveillance"). Outcomes and safety data for the strategies were based on the CLARINET trial and its extension. ${ }^{3,4}$ Patients began in the "not progressed" state and may remain so, experience disease progression, or die each month (Figure 1). After disease progression, patients began the next line of therapy in the following month. The subsequent therapy sequence was informed by NCCN Guidelines: ${ }^{177} \mathrm{Lu}-$ dotatate, everolimus, followed by sunitinib for patients with pancreatic NETs (Table 1$){ }^{2}$

\section{Model Calibration}

We determined rates of progression and preprogression and postprogression survival that produced OS and PFS curves consistent with trial data (Figure 2). We used a previously validated methodology to simulate individualpatient data for the OS and PFS curves. ${ }^{8}$ To represent the survival curves during the respective trial's study period and to extrapolate outcomes past the study period, we fit parametric functions to the OS and PFS curves in

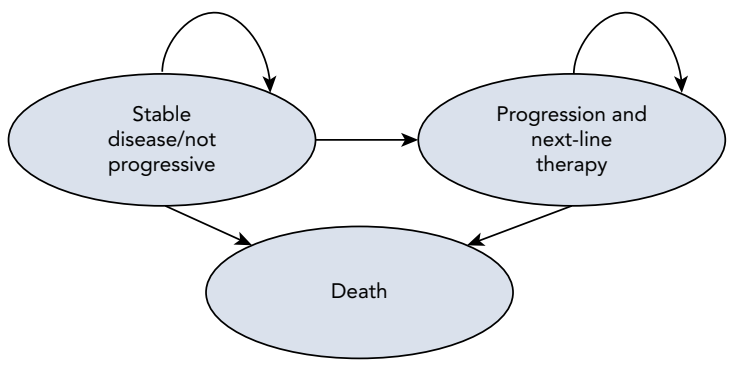

Figure 1. Diagram of the Markov model structure. Patients transition from the stable disease/not progressed disease state to progression or death based on transition probabilities derived from the CLARINET trial and the CLARINET extension., ${ }^{3,4}$ Therapy for progressed disease is outlined in Table 1. accordance with the UK's National Institute for Health and Care Excellence (NICE) recommendations. ${ }^{9}$ Goodnessof-fit criteria, plausibility of extrapolation, and visual fit were used to choose the parametric survival function in our base case (supplemental eTable 3, available with this article at JNCCN.org). Multiple sensitivity analyses were performed using a wide range of parametric fits (supplemental eTables 5 and 6) to assess the impact of extrapolation assumptions. For patients who underwent active surveillance and received lanreotide after disease progression, subsequent PFS was based on the CLARINET extension. ${ }^{4}$ We used an optimization-based algorithm programmed using MATLAB R2018b software (MathWorks) to solve for probabilities of progression and preprogression and postprogression mortality. We provide an alternative calibration to examine calibration's impact on the results in supplemental eAppendix 1 .

Progression rates while receiving subsequent therapies were modeled based on PFS curves from relevant trials (Table 1), whereas mortality rates were informed directly by the CLARINET trial. Sensitivity analyses explored the impact of progression rates on subsequent therapies.

Parametric regression was performed using the flexsurvreg function (flexsurv package version 1.1 in $\mathrm{R}$ version 3.3.2; R Core Team, R Foundation for Statistical Computing). We implemented our cost-effectiveness model using TreeAge Pro 2020 release 1.0 software (TreeAge Software). Our methods conform to Consolidated Health Economic Evaluation Reporting Standards $^{10}$ and the Second Panel on Cost-Effectiveness in Health and Medicine ${ }^{11}$ (supplemental eTables 16 and 17).

\section{Efficacy and Safety Data}

Efficacy data for upfront lanreotide, active surveillance, and lanreotide after progression were derived from the CLARINET trial and its extension. ${ }^{3,4}$ In the base case, we assumed mortality was equal for both treatment arms based on the CLARINET trial (log-rank test comparing OS curves; $P=.88$ ). This assumption was relaxed in scenario analyses (supplemental eTables 5 and 6).

Adverse effect (AE) rates were derived from CLARINET and the CLARINET extension (Table 2) and from trials outlined in Table 1 for relapsed disease therapy. Grades 3 and 4 AEs were included.

\section{Costs}

\section{Perspective}

We adopted a US Medicare healthcare sector perspective, including all healthcare costs borne by Medicare, Medicare supplemental insurance, and out-of-pocket costs. Costs and quality-adjusted life-years (QALYs) were discounted at 3\% annually. When necessary, costs were inflated to 2020 US dollars using the Personal Health 
Table 1. Sequence of Therapies Used in the Model, by Initial Therapy ${ }^{a}$

\begin{tabular}{|c|c|c|}
\hline & Upfront Lanreotide & Active Surveillance \\
\hline Third-line therapy & Everolimus $^{45}$ & ${ }^{177} \mathrm{Lu}$-dotatate with octreotide $\mathrm{LAR}^{44}$ \\
\hline Fifth-line therapy & Subsequent therapies/healthcare costs ${ }^{19}$ & Sunitinib for patients with cancer of pancreatic origin ${ }^{46}$ \\
\hline Sixth-line therapy & Subsequent healthcare costs ${ }^{19}$ & Subsequent healthcare costs ${ }^{19}$ \\
\hline
\end{tabular}

Abbreviation: LAR, long-acting release.

aThe references cited in the table refer to the trials that reported data on the progression probabilities while receiving therapy for relapsed disease.

Care Expenditure Index ${ }^{12,13}$ when available (through 2017), the Bureau of Economic Analysis personal consumption expenditure price index ${ }^{14}$ through 2019 per the Second Panel on Cost-Effectiveness in Health and Medicine, ${ }^{11}$ and the Consumer Price Index ${ }^{15}$ to inflate to 2020 US dollars.

\section{Drug Costs}

Lanreotide prices were based on the Medicare Part B payment allowance limit (ASP $+6 \%),{ }^{5}$ and administration costs were incorporated. ${ }^{177} \mathrm{Lu}$-dotatate prices were based on the Medicare Outpatient Prospective Payment System reimbursement rates. ${ }^{16}$ Everolimus and sunitinib were assumed to be paid through Medicare Part D, and drug costs were set to $64 \%$ of average wholesale price, based on the recommendations of the US Department of Veterans Affairs Health Economics Resource Center, which are informed by a US Congressional Budget Office report. $^{17,18}$ For upfront lanreotide, patients received $120 \mathrm{mg}$ every 28 days until progression. Patients treated with the active surveillance strategy received $120 \mathrm{mg}$ of lanreotide every 28 days starting the month after progression. Dosages for subsequent lines of therapy were based on relevant trials (Tables 1 and 2).

\section{AE Costs}

Each grade 3 and $4 \mathrm{AE}$ is assumed to result in a hospitalization incurring costs based on Medicare Severity Diagnosis Related Group payments. ${ }^{19}$

\section{Additional Healthcare Costs}

Additional healthcare costs are age-based per data published by Guy et al. ${ }^{20}$ Additional monitoring costs are based on NCCN recommendations. ${ }^{2}$

\section{Utilities}

Quality of life (QoL) weights for the base case are based on a publication by Swinburn et al, ${ }^{21}$ who developed clinical vignettes to describe preprogression and postprogression states for patients with NETs and developed utilities through interviews with the general population using the time trade-off method. ${ }^{22}$ We repeat the analysis
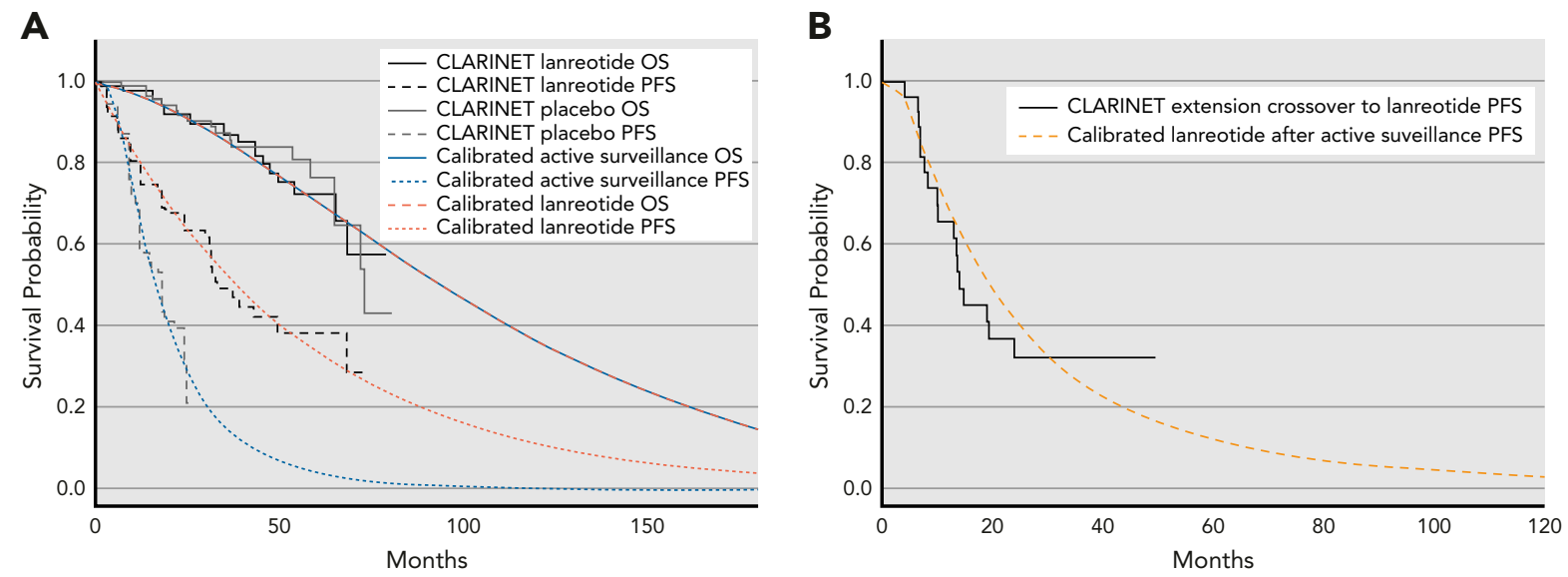

Figure 2. Survival curves. (A) Active surveillance and lanreotide survival curves. Modeled lanreotide and active surveillance OS and PFS curves used in the base case are compared with CLARINET trial curves from Caplin et al. 3,4 PFS data for the lanreotide arm have longer follow-up in the extension and thus were used for model calibration. (B) PFS for patients receiving lanreotide after progression while on active surveillance. PFS curves from the CLARINET trial extension ${ }^{4}$ are plotted with modeled PFS for patients receiving lanreotide after progression while on active surveillance.

Abbreviations: OS, overall survival; PFS, progression-free survival. 


\section{Table 2. Model Parameters Used for Base Case Analysis}

Distribution Used in PSA

\section{Health State Utilities (Yearly)}

\author{
Progression-free
}

Progressed disease

Death

Costs (USD)

\section{Lanreotide}

$\$ 7,638.48$ Not varied/inherent to perspective

Lanreotide injection

$\$ 14.44$ Not varied/inherent to perspective

${ }^{177}$ Lu-dotatate

$\$ 51,834$ Not varied/inherent to perspective

Octreotide LAR

$\$ 6,182.55$ Not varied/inherent to perspective

Everolimus

$\$ 13,105.32$ Not varied/inherent to perspective

Sunitinib

$\$ 13,489.88$ Not varied/inherent to perspective
Comments/Reference

Swinburn et $\mathrm{al}^{21}$

Swinburn et $\mathrm{al}^{21}$

Assumption/Convention
$\$ 489.97$
Per 120 mg (dose administered every $28 \mathrm{~d}$ ) Medicare Part B payment allowance limit (ASP plus $6 \%)^{5}$

Per every 28-d injection for lanreotide CMS physician fee schedule ${ }^{48}$ HCPCS code 96372; locality 0000000

Per 7,400 MBq. Medicare cost for HCPCS code A9513 ${ }^{16}$

Per $30 \mathrm{mg}$

Medicare Part B payment allowance limit (ASP plus $6 \%)^{5}$

$64 \%$ of average wholesale price ${ }^{49}$

Monthly cost for $10 \mathrm{mg} / \mathrm{d}^{\mathrm{a}}$

$64 \%$ of average wholesale price ${ }^{50}$ Monthly cost for $37.5 \mathrm{mg} / \mathrm{d}^{\mathrm{a}}$

Sum of costs outlined below

Expert opinion and NCCN Guidelines ${ }^{2}$

Incurred every 6 mo

Medicare clinical laboratory fee schedule ${ }^{51}$

Screening cost components

Comprehensive metabolic panel

$\$ 10.56$ Not varied/inherent to perspective

Medicare clinical laboratory fee schedule ${ }^{51}$

Complete blood count with differential

$\$ 7.77$ Not varied/inherent to perspective

Medicare clinical laboratory fee schedule ${ }^{51}$

Chromogranin A

$\$ 25.57$ Not varied/inherent to perspective

CT abdomen and pelvis with contrast

$\$ 332.39$ Not varied/inherent to perspective

$\$ 113.68$ Not varied/inherent to perspective

Physician office visit

Yearly additional healthcare

costs during therapy, total (USD)

$\$ 6,524.94$ Varied by component cost (listed below)

Ambulatory care

$\$ 4,558.5495 \% \mathrm{Cl}, 4,017.58-5,099.50$;

normal distribution: mean, 4,558.54; SD, 276

Other health service

$\$ 1,966.4095 \% \mathrm{Cl}, 1,641.04-2,291.76$.

normal distribution: mean, 1,966.40; SD, 166

Yearly costs after all

pharmaceutical treatment lines

$\$ 11,245.26$ Varied by component cost (listed below)

complete, total (USD)

\section{Ambulatory care}

$\$ 4,558.5495 \% \mathrm{Cl}, 4,017.58-5,099.50$

normal distribution: mean, 4,558.54; SD, 276

Other health service

$\$ 1,966.4095 \% \mathrm{Cl}, 1,641.04-2,291.76$;

normal distribution: mean, 1,966.40; SD, 166

Inpatient care
Sum of costs of inpatient care, ambulatory care, and "other health services" from Guy et al ${ }^{20}$ for patients aged $>65$ y, $\$ 9,615$ in 2010 US dollars, converted to 2020 US dollars for analysis

\author{
$\$ 4,720.3295 \% \mathrm{Cl}, 3,973.56-5,467.08$; \\ normal distribution: mean, 4,720.32; SD, 381
}


Table 2. Model Parameters Used for Base Case Analysis (cont.)

\begin{tabular}{|c|c|c|c|c|}
\hline & Lanreotide & $\begin{array}{l}\text { Active } \\
\text { Surveillance }\end{array}$ & $\begin{array}{l}\text { Lanreotide After } \\
\text { Progression on } \\
\text { Active } \\
\text { Surveillance }\end{array}$ & Comments/Reference \\
\hline Median time on therapy from respective trial & $24 \mathrm{mo}$ & $15 \mathrm{mo}$ & $13 \mathrm{mo}$ & \\
\hline Source of data & $\begin{array}{l}\text { Lanreotide arm } \\
\text { of CLARINET } \\
\text { trial; } \\
\text { Caplin et al }{ }^{3}\end{array}$ & $\begin{array}{l}\text { Placebo arm } \\
\text { of CLARINET } \\
\text { trial; } \\
\text { Caplin et al }{ }^{3}\end{array}$ & $\begin{array}{l}\text { Lanreotide after } \\
\text { progression on } \\
\text { placebo; } \\
\text { Caplin et } \mathrm{al}^{4}\end{array}$ & \\
\hline Percentage of patients experiencing SAE & $25 \%$ & $31 \%$ & $23 \%$ & $\begin{array}{l}\text { The rate of SAEs overall was reported, and separately the } \\
\text { rate of AEs of any grade was reported per event } \\
\text { We multiplied the SAE rate by the overall reported rate } \\
\text { per AE to get the final SAE rate used in the model }\end{array}$ \\
\hline $\begin{array}{l}\text { Percentage of patients experiencing } \\
\text { grade } 3 \text { or } 4 A E^{b}\end{array}$ & & & & $\begin{array}{l}\text { The final value used in the analysis was } S A E \text { percentage } \\
\text { times } A E \text {, converted from a rate over the course of } \\
\text { median time on therapy and converted to a monthly } \\
\text { probability }\end{array}$ \\
\hline Hyperglycemia & $5 \%$ & $0 \%$ & $0 \%$ & \\
\hline Nausea/Vomiting & $14 \%$ & $2 \%$ & $19 \%$ & \\
\hline Headache & $5 \%$ & $2 \%$ & $9 \%$ & \\
\hline Cholelithiasis & $10 \%$ & $3 \%$ & $4 \%$ & \\
\hline Abdominal pain & $14 \%$ & $2 \%$ & $13 \%$ & \\
\hline Hypertension & $0 \%$ & $0 \%$ & $11 \%$ & \\
\hline Constipation & $0 \%$ & $0 \%$ & $2 \%$ & \\
\hline Dizziness & $0 \%$ & $0 \%$ & $4 \%$ & \\
\hline \multicolumn{5}{|l|}{ Survival Curve Modeling } \\
\hline Progression-free survival & $\begin{array}{l}\text { Lanreotide after } \\
\text { progression on } \\
\text { placebo }\end{array}$ & Log-normal & $\begin{array}{l}\text { Mean: } 2.975 \\
\text { SD: } 0.963\end{array}$ & $\begin{array}{l}\text { SE mean } 0.205 \\
\text { SE SD: } 0.174 \\
\rho=0.302\end{array}$ \\
\hline \multicolumn{5}{|l|}{ Overall survival } \\
\hline \multirow[t]{2}{*}{ Base case (same rate of mortality assumed) } & Lanreotide & Weibull & $\begin{array}{l}\text { Shape: } 1.55 \\
\text { Scale: } 120.47\end{array}$ & $\begin{array}{l}\text { SE shape: } 0.3 \\
\text { SE scale: } 26.91 \\
\rho=-0.762\end{array}$ \\
\hline & $\begin{array}{l}\text { Active } \\
\text { surveillance }\end{array}$ & Weibull & $\begin{array}{l}\text { Shape: } 1.55 \\
\text { Scale: } 120.47\end{array}$ & $\begin{array}{l}\text { SE shape: } 0.3 \\
\text { SE scale: } 26.91 \\
\rho=-0.762\end{array}$ \\
\hline
\end{tabular}

Abbreviations: $\mathrm{AE}$, adverse effect; ASP, average sales price; CMS, Centers for Medicare and Medicaid Services; HCPCS, Healthcare Common Procedural Coding System; PSA, probabilistic sensitivity analysis; SAE, serious adverse effect.

aDays per month: 30.42 .

bAdditional information on distributions used in the PSA available in Supplemental eTable 7.

'Parameters drawn from normal distributions with SDs equivalent to listed standard errors and correlations between parameters defined by correlation coefficients ( $\rho$ ).

'Based on CLARINET placebo.

using data published by Meng et al, ${ }^{23}$ who analyzed data collected from patients during the CLARINET trial using the EORTC Quality-of-Life Questionnaire ${ }^{24}$ and a mapping algorithm to produce EuroQol 5 dimensions-based QoL weights. ${ }^{25}$ The data from Swinburn et $\mathrm{al}^{21}$ were chosen for the base case because the Meng et $\mathrm{al}^{23}$ data for the progressed state were based on QoL surveys completed only at a visit 1 month after progression (no subsequent 
QoL data were collected in the CLARINET trial), and therefore they may not be representative of the average QoL experienced while in the progressed state. The QoL weight for the "not progressed" state was similar between Swinburn and Meng data ( 0.771 vs 0.776 , respectively), but the weight for the progressed state was much lower ( 0.612 vs 0.726 , respectively). Using Swinburn data thus resulted in a greater penalty for progression, favoring the lanreotide arm. Results from additional models using the Meng QoL data are presented in supplemental eTable 5. Each AE incurs a utility decrement based on the expected duration of the $\mathrm{AE}$ subtracted from the baseline utility (supplemental eTables 7 and 8).

\section{Sensitivity Analyses}

One-way sensitivity analyses were performed on key parameters to determine the impact on our results. We performed extensive sensitivity analyses on survival curve extrapolation by producing 28 different models using 2 calibration methods with various combinations of extrapolated survival curves including scenarios in which OS differed between treatment strategies (supplemental eTables 5 and 6). A probabilistic sensitivity analysis with 10,000 Monte Carlo simulations was performed to determine the effect of uncertainty around model parametric estimates.

\section{Results}

In the modeled cohort, the projected median PFS in the upfront lanreotide arm and active surveillance arm were 38.5 and 16.5 undiscounted months, respectively. The projected median OS for both arms was 95 undiscounted months. Supplemental eTable 1 lists the percentage of patients reaching the various lines of therapy and time spent in each line of therapy.

The combination of modeled survival and progression benefits led to 5.21 discounted QALYs (8.98 undiscounted years; 7.50 discounted years) at $\$ 804,600$ for the upfront lanreotide arm and 4.84 QALYs (8.98 undiscounted years; 7.50 discounted years) at $\$ 590,200$ for the active surveillance arm (Table 3). The upfront lanreotide arm cost $\$ 578,500 /$ QALY gained compared with the active surveillance arm. If the price of lanreotide were decreased to match that of octreotide long-acting release (LAR), upfront lanreotide would cost \$482,700/ QALY gained.

\section{Sensitivity Analysis}

Because of the uncertainty in extrapolating survival curves beyond the study period, we performed extensive analyses using various combinations of parametric forms to model PFS and OS of the patients undergoing active surveillance, those receiving upfront lanreotide, and those who cross over to lanreotide after progression on active surveillance. We also provide results for an alternative method of calibration (supplemental eAppendix 1). The incremental costeffectiveness ratios (ICERs) range from $\$ 373,000$ to $\$ 581,600$ per QALY gained for the models assuming equal OS between the 2 treatment arms. When upfront lanreotide was assumed to have improved OS compared with active surveillance, the ICERs ranged from $\$ 214,000$ to $\$ 458,300$ per QALY gained.

Based on our analyses, the ICER is most sensitive to the price of lanreotide. Substantial decreases in the price of lanreotide would improve its economic value compared with active surveillance followed by lanreotide. Lowering the price of lanreotide by $96 \%$ to $\$ 370$ per $120 \mathrm{mg}$ and by $85 \%$ to $\$ 1,130$ per $120 \mathrm{mg}$ would allow the upfront lanreotide strategy to meet willingness-topay (WTP) thresholds of $\$ 100,000 /$ QALY gained and $\$ 150,000 /$ QALY gained, respectively. This price is lower than publicly available prices for federal pharmaceutical purchasers (Figure 3A). ${ }^{26}$ Across the extrapolation scenarios, the price of lanreotide would have to be lowered to $\$ 370$ to $\$ 4,000$ or $\$ 1,130$ to $\$ 5,600$ per $120 \mathrm{mg}$ to meet a WTP threshold of $\$ 100,000 /$ QALY gained or $\$ 150,000 /$ QALY gained, respectively. Across all extrapolation scenarios in which OS is assumed to be equivalent between strategies, lanreotide's cost would need to be decreased to $<\$ 3,200$ or $<\$ 4,000$ per $120 \mathrm{mg}$ to cost less than $\$ 100,000 /$ QALY gained or $\$ 150,000 /$ QALY gained, respectively; these results are derived from models that produce results with upper bounds on cost of lanreotide to meet these WTP thresholds (see "Model Calibration Methods" in supplemental eAppendix 1).

\section{Table 3. Cost-Effectiveness Analysis Results From Base Case}

\begin{tabular}{|lccccr|}
\hline Strategy & Cost (Discounted) & Incremental Cost & QALYs (Discounted) & Incremental QALYs & ICER \\
\hline $\begin{array}{l}\text { Active surveillance and lanreotide } \\
\text { after progression }\end{array}$ & $\$ 590,189$ & & 4.84 & \\
\hline Lanreotide as initial therapy & $\$ 804,563$ & $\$ 214,374$ & 5.21 & 0.37 \\
\hline
\end{tabular}

These data compare active surveillance followed by lanreotide as second-line therapy with lanreotide as first-line therapy. Lanreotide as first-line therapy was modeled to cost an additional $\$ 214,374$ in healthcare costs and add an additional 0.37 QALYs as compared with active surveillance, which assumes lanreotide as second-line therapy. Costs are derived from Medicare perspective and include additional healthcare costs.

Abbreviations: ICER, incremental cost-effectiveness ratio; QALY, quality-adjusted life-year. 
A

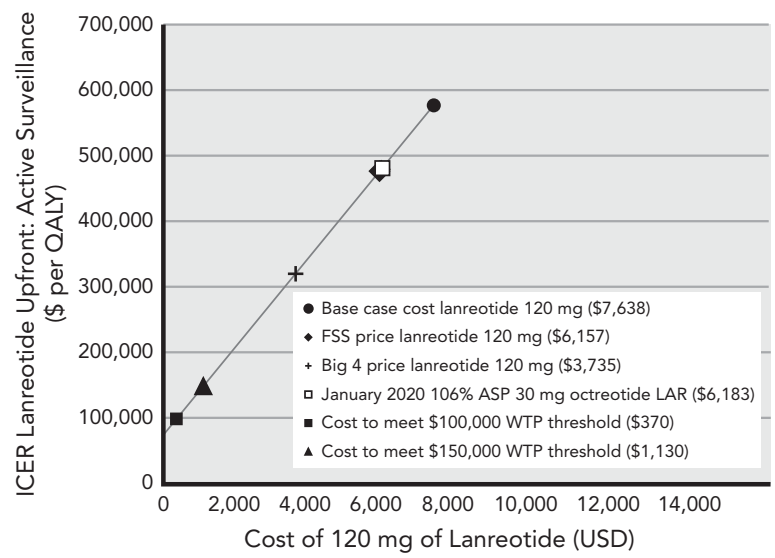

C

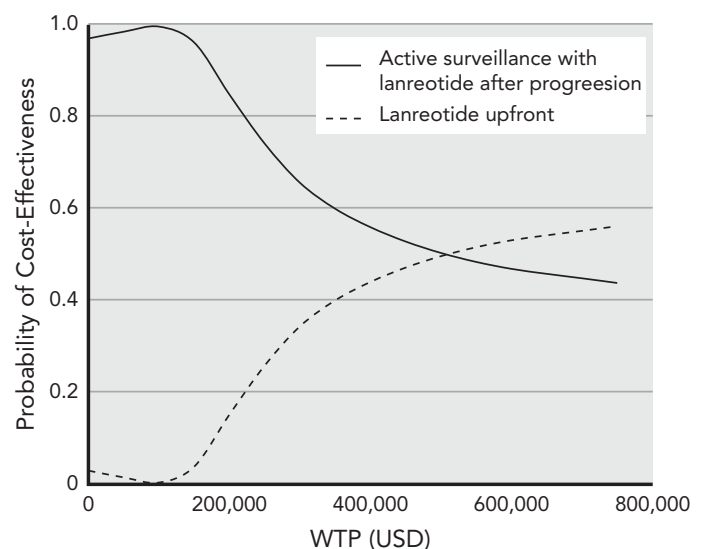

B

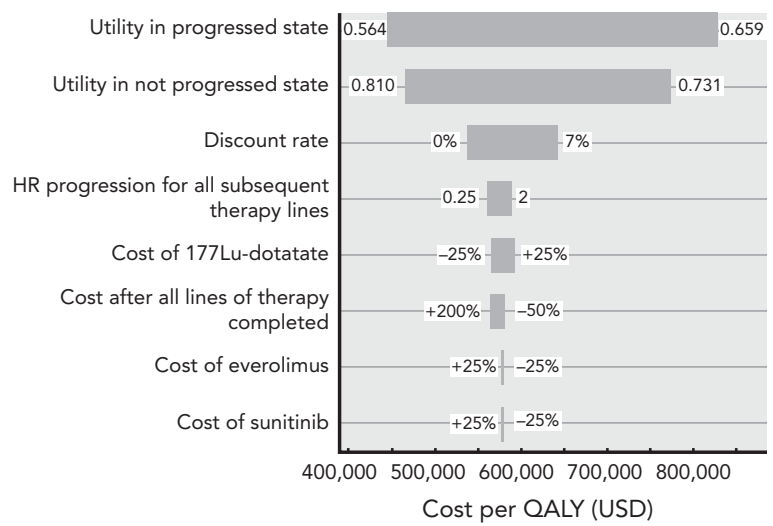

D

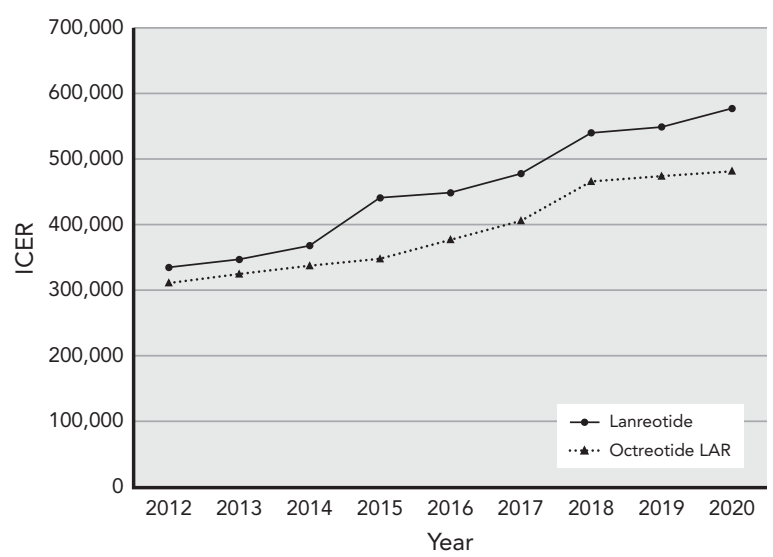

Figure 3. Sensitivity analyses. (A) Sensitivity to cost of $120 \mathrm{mg}$ of lanreotide. The base case $(\$ 7,638)$ is plotted together with other benchmarks, such as the FSS and Big 4 prices. The current cost to Medicare of $30 \mathrm{mg}$ of octreotide LAR $(\$ 6,138)$ is also highlighted. At costs above the WTP threshold, active surveillance is the preferred strategy. (B) One-way sensitivity analysis of various parameters. The ICER is sensitive to changes in the utilities for the progressed and not progressed states; however, over the $95 \% \mathrm{Cls}$ for the utilities used in the analysis, those elicited by Swinburn et al, ${ }^{21}$ the ICER remains above $\$ 350,000 /$ QALY gained. (C) PSA results (cost-effectiveness acceptability curve). In the base case, upfront lanreotide is the preferred strategy in $0.4 \%$ of iterations for a $\$ 100,000 / \mathrm{QALY}$ WTP threshold and 3.9\% of iterations for a \$150,000/QALY WTP threshold. (D) Modeled ICERs over time for lanreotide and octreotide LAR. The ICER increases since 2012 are largely caused by increases in the costs of lanreotide and octreotide LAR, because the cost of lanreotide increased from $\$ 3,670$ per 120 mg in January 2012 ( $\$ 4,470$ in 2020 US dollars) to $\$ 7,638$ per $120 \mathrm{mg}$ in January 2020 , and the cost of octreotide LAR increased from $\$ 3,650$ per 30 mg in January 2012 ( $\$ 4,100$ in 2020 US dollars) to $\$ 6,200$ per $30 \mathrm{mg}$ in January 2020 (ASP plus 6\%). For this analysis, ${ }^{177} \mathrm{Lu}$-dotatate is included as a line of therapy starting in 2018 and excluded before then. When necessary, costs were inflated to 2020 US dollars.

Abbreviations: ASP, average sales price; Big 4 price, price available to 4 largest federal purchasers of pharmaceuticals: US Department of Veterans Affairs, US Department of Defense, US Coast Guard, and US Public Health Service; HR, hazard ratio; FSS, Federal Supply Schedule; ICER, incremental cost-effectiveness ratio; LAR, long-acting release; PSA, probabilistic sensitivity analysis; QALY, quality-adjusted life-year; WTP, willingness to pay.

In one-way sensitivity analyses, varying utilities for health states, costs of subsequent therapies, and costs after the modeled lines of therapy did not alter our conclusions (Figure 3B). Across these analyses, the upfront lanreotide arm cost $>\$ 350,000 /$ QALY gained compared with the active surveillance arm.

Using Meng et $\mathrm{al}^{23}$ utilities, QoL in the progressed state is modeled to be better $(0.726$ vs 0.612 from Swinburn); thus, progression is less negatively impactful. Assuming utilities from Meng et al, the economic value of upfront lanreotide is substantially worse $(\$ 1,837,000 /$ QALY) than the base case.
The cost of lanreotide has increased over time, from $\$ 3,970$ per $120 \mathrm{mg}$ in January 2012 ( $\$ 4,470$ in 2020 US dollars) to $\$ 7,638$ per $120 \mathrm{mg}$ in January $2020 .{ }^{27}$ Figure 3C shows the modeled increase in ICER over this time period, from $\$ 336,100 /$ QALY gained in 2012 to $\$ 578,600 /$ QALY gained in 2020 (in 2020 US dollars).

Although the main results of the analysis are not sensitive to the costs of ${ }^{177} \mathrm{Lu}$-dotatate, everolimus, and sunitinib, their costs do impact the price at which lanreotide becomes cost-effective. For our base case, with lower costs of ${ }^{177} \mathrm{Lu}$-dotatate, sunitinib, and everolimus, the price of lanreotide at which upfront lanreotide becomes 
cost-effective is higher. For our base case analysis, if ${ }^{177} \mathrm{Lu}$-dotatate, everolimus, and sunitinib costs are decreased by $75 \%$, lanreotide is cost-effective at $\$ 880$ and $\$ 1,640$ per $120 \mathrm{mg}$ for the $\$ 100,000 /$ QALY gained and $\$ 150,000 /$ QALY gained WTP thresholds, respectively (supplemental eTable 13). In a probabilistic sensitivity analysis with 10,000 samples, upfront lanreotide was cost-effective at a WTP of $\$ 100,000 /$ QALY gained and $\$ 150,000 /$ QALY gained in $0.4 \%$ and $3.9 \%$ of samples, respectively (Figure 3C).

\section{Discussion}

Cancer is among the costliest chronic medical conditions in the United States, ${ }^{28}$ and costs have continued to increase, with a projected increase of $40 \%$ from 2010 to $2020 .{ }^{29}$ Drug costs paid through Medicare Part B have increased from $\$ 13.4$ billion in 2005 to $\$ 29.1$ billion in 2016 , reflecting an annualized increase of $7.4 \%,{ }^{30}$ outpacing gross domestic product growth (3.7\% annualized over this same period). ${ }^{31}$ As of 2014, cancer drug costs represented $42 \%$ of Medicare Part B drug costs. ${ }^{32}$ Because of concerns related to the economic impact of the increasing cost of cancer care, there has been recent emphasis on quantifying cost and value. ${ }^{7,33,34}$ NETs are increasing in incidence and prevalence, with a higher prevalence than esophageal cancer and pancreatic adenocarcinoma combined, ${ }^{35}$ representing a bigger public health problem than previously recognized. In addition, patients with NETs have long life expectancy compared with those with other cancer types, with an estimated median survival of 9.3 years. ${ }^{35}$ Shen et $\mathrm{al}^{36}$ previously reported relatively high 5-year costs of care for patients with NETs, thought to be due largely to high costs in the continuing phase of care and longer survival compared with other cancers. Recognition of this illustrates that it is important to study the value of therapy for NETs and all chronic cancers.

Our study shows that, among patients with grades 1 and 2 nonfunctioning gastroenteropancreatic NETs, upfront lanreotide treatment likely results in QoL gains compared with active surveillance followed by lanreotide after progression. However, at current prices, it would not be cost-effective at conventional WTP thresholds. For our base case analysis, the ICER is \$578,500/QALY gained.

Based on the CLARINET trial, lanreotide was approved by the FDA in 2014 for the treatment of patients with unresectable, well or moderately differentiated, locally advanced or metastatic gastroenteropancreatic NETs to prolong PFS. ${ }^{3}$ Patients whose disease progressed on placebo and who crossed over to lanreotide achieved stabilization of their disease and experienced a median of 14 months of PFS in the CLARINET extension. ${ }^{4}$
Although lanreotide and octreotide LAR have never been directly compared for this indication, both are given equal recommendation in the NCCN Guidelines. ${ }^{2}$ NCCN Guidelines recommend a somatostatin analogue (lanreotide or octreotide) up front or active surveillance with somatostatin analogue upon progression as a first-line therapy option for gastrointestinal NETs and as a consideration for pancreatic NETs. ${ }^{2}$

The recently adopted NCCN Evidence Blocks provide guidance on the "affordability" (projected overall cost) of a given treatment course; they do not directly evaluate its value (whether its benefits justify the costs). ${ }^{7}$ Affordability ratings are based on expert panel members' knowledge of costs associated with the treatment regimen (efficacy and safety are evaluated similarly as separate dimensions), and Carlson and Jonasch ${ }^{7}$ indicated that the methodology to determine affordability is still a work in progress. Current NCCN Evidence Blocks designate lanreotide and octreotide as "expensive" treatment options, assigning a 2 on a scale from 1 through 5 (with 1 being least affordable). ${ }^{2}$ Our study is the first published analysis to directly address the value of upfront lanreotide compared with active surveillance followed by lanreotide. The base case and extensive sensitivity analyses show that although upfront lanreotide may provide an increase in QALYs over active surveillance followed by lanreotide upon progression, at its current Medicare price, its value is poor for this indication-it costs approximately 3 to 5 times the often-cited WTP thresholds for the United States. ${ }^{37,38}$ Upfront lanreotide would be cost-effective at conventional WTP thresholds with considerable price reductions $(96 \%$ and $85 \%$ decreases to meet $<\$ 100,000 /$ QALY gained and $<\$ 150,000 /$ QALY gained thresholds, respectively).

It has been noted that postlaunch prices of individual injectable cancer drugs have increased substantially over time. ${ }^{39}$ Lanreotide has experienced significant price increases in recent years. We examined the value impact of this price increase on the ICER from 2012 through 2020 (Figure 3C). Although it never would have met commonly cited WTP thresholds during this period, the increase in cost has made upfront lanreotide an increasingly poor value.

Our study has some important limitations. We limited our analysis to examine only strategies that incorporate lanreotide as either first-line or second-line therapy, and therefore, we did not evaluate strategies that do not consider lanreotide for therapy in this population; the ICERs and prices at which lanreotide meets the reported WTP thresholds must be interpreted within this context. We believe this approach is justified because these are the 2 strategies recommended by the NCCN Guidelines for this population. Extrapolation beyond the 
study period for which reported data exist is a challenge for all cost-effectiveness analyses. To address this, we present the results of multiple models that have varying assumptions about extrapolation of PFS and OS. The overall results are not sensitive to the extrapolation assumptions; however, the cost at which lanreotide becomes cost-effective does vary. Other potential limitations result from the use of the placebo arm to model active surveillance. The QoL measurements used (Swinburn et $\mathrm{al}^{21}$ and Meng et $\mathrm{al}^{23}$ ) do not incorporate a decrement associated with anxiety due to lack of treatment, which may result in a slight overestimate of utilities for the preprogression state in the active surveillance arm of our analysis. However, unrealistically large decreases in the preprogression utility to far below the utility of the progressed state would be needed to alter the conclusion of our analysis (supplemental eFigure 9). Other potential placebo effects, outlined by Hawkins and Scott, ${ }^{40}$ for which we are unable to account may either increase or decrease the ICER. However, use of the placebo arm for the active surveillance comparator agrees with the manufacturer's health technology assessment submitted to Australia's Pharmaceutical Advisory Committee. ${ }^{41}$ In addition, we did not model the expiration of patents for lanreotide. Lanreotide currently has active patents and marketing exclusivity in the United States. ${ }^{42} \mathrm{We}$ adopted the position of the Institute for Clinical and Economic Review ${ }^{43}$ that it is difficult to predict future prices of drugs when coming off patent because of unpredictable price trajectory before patent expiration and other factors. We present the ICER if lanreotide were priced similarly to octreotide LAR, which has no active patent protections (see Figure $3 \mathrm{~A}$ ) ${ }^{44}$; at this price, it would also not be cost-effective at a WTP threshold of $\$ 100,000$ or $\$ 150,000$ per QALY gained.

\section{Conclusions}

At its current price, our analysis indicates that lanreotide is not cost-effective as initial therapy for patients with metastatic enteropancreatic NETs and that it should be reserved for postprogression treatment. Treatments that offer appreciable clinical benefit but that are not deemed cost-effective put practicing oncologists in a difficult position even if guidelines recommend against them. The importance of further innovation in pricing for value is highlighted by such situations. We do not recommend that this study be used in isolation to make treatment recommendations for patients with NETs; instead, we advise that it be viewed in conjunction with other available evidence to inform updated practice guidelines going forward and as part of the discussion in working toward high-value care and an economically sustainable healthcare system.

Submitted October 31, 2019; accepted for publication March 23, 2020.

Previous presentation: This study was presented as an abstract and poster at the North American Neuroendocrine Tumor Society (NANETS) Annual Multidisciplinary NET Disease Symposium; October 6, 2018; Seattle, Washington. Abstract 114

Author contributions: Study concept and design: All authors. Data collection and assembly: Barnes, Lin, Gupta, Kunz. Data analysis and interpretation: Barnes, Lin, Owens, Goldhaber-Fiebert, Kunz. Manuscript preparation: All authors.

Disclosures: Dr. Kunz has disclosed that she receives grant/research suppor from Advanced Accelerator Applications, Ipsen, Lexicon, Xencor, and B.R.A.H.M.S., and that she is a scientific advisor for Advanced Accelerator Applications. The remaining authors have disclosed that they have not received any financial consideration from any person or organization to support the preparation, analysis, results, or discussion of this article.

Funding: Drs. Barnes and Lin were supported by funding from the Office of Academic Affiliations, Department of Veterans Affairs, Advanced Fellowship in HSR\&D. Dr. Owens was supported by the Department of Veterans Affairs. All views expressed in this article are those of the authors and do not necessarily reflect the views of the Department of Veterans Affairs.

Correspondence: James I. Barnes, MD, MS, Center for Primary Care and Outcomes Research/Center for Health Policy, Department of Medicine, Stanford University School of Medicine, 117 Encina Commons, Stanford, CA 94305. Email: jibarnes@stanfordalumni.org

\section{References}

1. Dasari A, Shen C, Halperin D, et al. Trends in the incidence, prevalence, and survival outcomes in patients with neuroendocrine tumors in the United States. JAMA Oncol 2017;3:1335-1342.

2. Shah MH, Goldner WS, Benson AB III, et al. NCCN Clinical Practice Guidelines in Oncology: Neuroendocrine and Adrenal Tumors. Version 1.2019. Accessed March 18, 2020. To view the most recent version, visit NCCN.org

3. Caplin ME, Pavel M, Ćwikła JB, et al. Lanreotide in metastatic enteropancreatic neuroendocrine tumors. N Engl J Med 2014;371:224-233.

4. Caplin ME, Pavel M, Ćwikła JB, et al. Anti-tumour effects of lanreotide for pancreatic and intestinal neuroendocrine tumours: the CLARINET openlabel extension study. Endocr Relat Cancer 2016;23:191-199.

5. Centers for Medicare and Medicaid Services. Medicare part B drug average sales price (ASP): 2020 ASP drug pricing files. Accessed January 20, 2020. Available at: https://www.cms.gov/medicare/medicare-partb-drug-average-sales-price/2020-asp-drug-pricing-files

6. Centers for Medicare and Medicaid Services. Medicare part B drug average sales price (ASP): 2009 ASP drug pricing files. Accessed March 25, 2019. Available at: https://www.cms.gov/Medicare/Medicare-Fee-for-

Service-Part-B-Drugs/McrPartBDrugAvgSalesPrice/01a1_2009aspfiles. html

7. Carlson RW, Jonasch E. NCCN Evidence Blocks. J Natl Compr Canc Netw 2016;14(Suppl 5):616-619

8. Guyot P, Ades AE, Ouwens MJNM, et al. Enhanced secondary analysis of survival data: reconstructing the data from published Kaplan-Meier survival curves. BMC Med Res Methodol 2012;12:9.

9. Latimer N. NICE DSU technical support document 14: survival analysis for economic evaluations alongside clinical trials - extrapolation with patientlevel data. Accessed November 20, 2018. Available at: http://nicedsu. org.uk/wp-content/uploads/2016/03/NICE-DSU-TSD-Survival-analysis. updated-March-2013.v2.pdf

10. Husereau D, Drummond M, Petrou S, et al. Consolidated Health Economic Evaluation Reporting Standards (CHEERS)—explanation and elaboration: a report of the ISPOR Health Economic Evaluation Publication Guidelines Good Reporting Practices Task Force. Value Health 2013;16:231-250.

11. Basu A. Cost-effectiveness in health and medicine. In: Neumann PJ, Sanders GD, Russell LB, et al, eds. Cost-Effectiveness in Health and Medicine, 2nd ed. New York, NY: Oxford University Press; 2017:201-235. 
12. Agency for Healthcare Research and Quality (AHRQ). Medical expenditure panel survey (MEPS): using appropriate price indices for analyses of health care expenditures or income across multiple years. Accessed December 8, 2018. Available at: https://meps.ahrq.gov/about_meps/ Price_Index.shtml\#a3

13. National Health Expenditure Accounts: Methodology Paper, 2018. Accessed August 17, 2020. Available at: https://www.cms.gov/files/ document/definitions-sources-and-methods.pdf

14. Federal Reserve Bank of St. Louis, FRED Economic Data. Personal consumption expenditures: services: health care (chain-type price index). Accessed December 12, 2018. Available at: https://fred.stlouisfed.org/ series/DHLCRG3A086NBEA

15. U.S. Bureau of Labor Statistics. Consumer price index (CPI). Accessed January 15, 2020. Available at: https://www.bls.gov/cpi/

16. Centers for Medicare and Medicaid Services. Medicare outpatient prospective payment system (OPPS) addendum B. Accessed February 25, 2020. Available at: https://www.cms.gov/Medicare/Medicare-Fee-forService-Payment/HospitalOutpatientPPS/Addendum-A-and-AddendumB-Updates-Items/2019-Oct-Addendum-B

17. U.S. Department of Veterans Affairs, Health Economics Resource Center (HERC). Determining the cost of pharmaceuticals for a cost-effectiveness analysis. Accessed January 9, 2017. Available at: http://www.herc. research.va.gov/include/page.asp?id=pharmaceutical-costs

18. Congress of the United States, Congressional Budget Office. Prices for brand-name drugs under selected federal programs. Accessed January 10, 2017. Available at: https://www.cbo.gov/sites/default/files/cbofiles/ ftpdocs/64xx/doc6481/06-16-prescriptdrug.pdf

19. Centers for Medicare and Medicaid Services. National summary of inpatient charge data by Medicare severity diagnosis related group (MSDRG), FY2015. Accessed February 8, 2019. Available at: https://data.cms. gov/Medicare-Inpatient/National-Summary-of-Inpatient-Charge-Databy-Medic/efwk-h4x3

20. Guy GP Jr, Ekwueme DU, Yabroff KR, et al. Economic burden of cancer survivorship among adults in the United States. J Clin Oncol 2013;31: 3749-3757.

21. Swinburn $P$, Wang J, Chandiwana $D$, et al. Elicitation of health state utilities in neuroendocrine tumours. J Med Econ 2012;15:681-687.

22. Torrance GW, Thomas WH, Sackett DL. A utility maximization model for evaluation of health care programs. Health Serv Res 1972;7:118-133.

23. Meng Y, McCarthy G, Berthon A, et al. Patient-reported health state utilities in metastatic gastroenteropancreatic neuroendocrine tumours-an analysis based on the CLARINET study. Health Qual Life Outcomes 2017;15:131

24. EORTC Quality of Life. Quality of life of cancer patients. Accessed March 26, 2019. Available at: https://qol.eortc.org/questionnaire/eortc-qlq-c30

25. McKenzie L, van der Pol M. Mapping the EORTC QLQ C-30 onto the EQ-5D instrument: the potential to estimate QALYs without generic preference data. Value Health 2009;12:167-171.

26. U.S. Department of Veterans Affairs. VA national acquisition center contract catalog search tool (CCST). Accessed January 16, 2019. Available at: https://www.va.gov/nac/Pharma/List?cboContractNumbers= \&cboContractorNames $=\&$ txtCriteria $1=$ Lanreotide\&TxtNDC $=$ \&txtPackage $=\& \mathrm{cboVAClass}=\&$ Sort $=1$ \&search $=$ Search

27. Centers for Medicare and Medicaid Services. Medicare part B drug average sales price. Accessed January 29, 2020. Available at: https:// www.cms.gov/Medicare/Medicare-Fee-for-Service-Part-B-Drugs/ McrPartBDrugAvgSalesPrice/index.html

28. Soni A. The five most costly conditions, 1996 and 2006: estimates for the U.S. civilian noninstitutionalized population. Statistical Brief \#248. Rockville, MD: Agency for Healthcare Research and Quality; 2009.

29. Mariotto $A B$, Yabroff $K R$, Shao $Y$, et al. Projections of the cost of cancer care in the United States: 2010-2020. J Natl Cancer Inst 2011;103:117-128.

30. Medicare Payment Advisory Commission. A data book: health care spending and the Medicare program. Accessed March 25, 2019. Available at: http://www.medpac.gov/docs/default-source/data-book/ jun18_databookentirereport_sec.pdf
31. U.S. Bureau of Economic Analysis, Gross Domestic Product [GDP], retrieved from FRED, Federal Reserve Bank of St. Louis. Accessed March 25, 2019. Available at: https://fred.stlouisfed.org/series/GDP

32. Sheingold S, Marchetti-Bowick E, Nguyen N, et al. Medicare part B drugs: pricing and incentives. Acccessed July 10, 2020. Available at: https:// aspe.hhs.gov/system/files/pdf/187581/PartBDrug.pdf

33. Schnipper LE, Davidson NE, Wollins DS, et al. American Society of Clinical Oncology statement: a conceptual framework to assess the value of cancer treatment options. J Clin Oncol 2015;33:2563-2577.

34. Schnipper LE, Davidson NE, Wollins DS, et al. Updating the American Society of Clinical Oncology value framework: revisions and reflections in response to comments received. J Clin Oncol 2016;34:2925-2934.

35. Kunz PL. Understanding neuroendocrine tumors-a NET gain. JAMA Oncol 2017;3:1343-1344.

36. Shen C, Dasari A, Gu D, et al. Costs of cancer care for elderly patients with neuroendocrine tumors. Pharmacoeconomics 2018;36:1005-1013.

37. Neumann PJ, Cohen JT, Weinstein MC. Updating cost-effectiveness-the curious resilience of the \$50,000-per-QALY threshold. N Engl J Med 2014; 371:796-797.

38. Woods B, Revill P, Sculpher M, et al. Country-level cost-effectiveness thresholds: initial estimates and the need for further research. Value Health 2016;19:929-935.

39. Gordon N, Stemmer SM, Greenberg D, et al. Trajectories of injectable cancer drug costs after launch in the United States. J Clin Oncol 2018;36: 319-325.

40. Hawkins N, Scott DA. Cost-effectiveness analysis: discount the placebo at your peril. Med Decis Making 2010;30:536-543.

41. Australian Government Department of Health. Pharmaceutical Benefits Scheme. Lanreotide: $120 \mathrm{mg}$ injection, Somatuline. Accessed May 24, 2019. Available at: https://www.pbs.gov.au/info/industry/listing/elements/pbac-meetings/psd/2015-11/lanreotide-somatuline-psd-11-2015

42. US Food and Drug Administration. Orange Book: approved drug products with therapeutic equivalence evaluations. Patent and exclusivity for N022074. Lanreotide Acetate (Somatuline Depot). Accessed February 11, 2019. Available at: https://www.accessdata.fda.gov/scripts/cder/ob/ patent_info.cfm?Product_No=001\&Appl_No=022074\&Appl_type $=\mathrm{N}$

43. Institute for Clinical and Economic Review (ICER). Final value assessment framework for 2017-2019. Accessed January 16, 2019. Available at: https://icer-review.org/final-vaf-2017-2019/

44. US Food and Drug Administration. Orange Book: approved drug products with therapeutic equivalence evaluations. Patent and exclusivity for N021008. Octreotide Acetate (Sandostatin LAR). Accessed February 11, 2019. Available at: https://www.accessdata.fda.gov/scripts/cder/ob/ patent_info.cfm?Product_No $=001 \&$ Appl_No $=021008 \&$ Appl_type $=\mathrm{N}$

45. Strosberg J, El-Haddad G, Wolin E, et al. Phase 3 trial of ${ }^{177}$ Lu-Dotatate for midgut neuroendocrine tumors. N Engl J Med 2017;376:125-135.

46. Yao JC, Shah MH, Ito $T$, et al. Everolimus for advanced pancreatic neuroendocrine tumors. N Engl J Med 2011;364:514-523.

47. Raymond E, Dahan L, Raoul JL, et al. Sunitinib malate for the treatment of pancreatic neuroendocrine tumors. N Engl J Med 2011;364:501-513.

48. Centers for Medicare and Medicaid Services. Physician Fee Schedule Search Tool. Accessed June 19, 2018. Available at: https://www.cms.gov/ apps/physician-fee-schedule/search/search-criteria.aspx

49. IBM MICROMEDEX. Everolimus pricing. Accessed May 17, 2017. Available at: http://www.micromedexsolutions.com/micromedex2/ librarian/PFActionld/evidencexpert.ShowRedBookSearchResults ForActivelngredient? SearchTerm = Everolimus\&navResults = relatedProductLookupRedBook

50. IBM MICROMEDEX. Sunitinib pricing. Accessed May 17, 2017. Available at: http://www.micromedexsolutions.com/micromedex2/librarian/PFActionld/evidencexpert.ShowRedBookSearchResultsForActivelngredient? SearchTerm =Sunitinib

51. Centers for Medicare and Medicaid Services. Clinical laboratory fee schedule 2018. Accessed January 22, 2019. Available at: https://www. cms.gov/Medicare/Medicare-Fee-for-Service-Payment/ClinicalLabFeeSched/ Clinical-Laboratory-Fee-Schedule-Files-Items/18CLAB.html? DLPage $=1 \& D L E n t r i e s=10 \& D L S o r t=2 \& D L S o r t D i r=$ descending 